\title{
Gear Fault Diagnosis Based on Empirical Mode Decomposition and 1.5 Dimension Spectrum
}

\author{
Jianhua Cai and Xiaoqin Li \\ Department of Physics and Electronics, Hunan University of Arts and Science, Changde 415000, China \\ Correspondence should be addressed to Jianhua Cai; cjh1021cjh@163.com
}

Received 20 July 2015; Revised 8 November 2015; Accepted 15 November 2015

Academic Editor: Didier Rémond

Copyright (C) 2016 J. Cai and X. Li. This is an open access article distributed under the Creative Commons Attribution License, which permits unrestricted use, distribution, and reproduction in any medium, provided the original work is properly cited.

\begin{abstract}
Aiming at the nonlinear and nonstationary feature of mechanical fault vibration signal, a new fault diagnosis method, which is based on a combination of empirical mode decomposition (EMD) and 1.5 dimension spectrum, is proposed. Firstly, the vibration signal is decomposed by EMD and the correlation coefficient between each intrinsic mode function and original signal is calculated. Then these intrinsic mode function components, which have a big correlation coefficient, are selected to estimate its 1.5 dimension spectrum. And this method uses 1.5 dimension spectrum of each intrinsic mode function to reconstruct its power spectrum. And these power spectrums are summed to obtain the primary power spectrum of gear fault signal. Finally, the information feature of fault is extracted from the reconstructed 1.5 dimension spectrum. A model to reconstruct 1.5 dimension spectrum is established, and the principle and steps of the method are presented. Some simulated and measured gear fault signals have been processed to demonstrate the effectiveness of new method. The result shows that this method can greatly inhibit the interference of Gauss noise to raise the SNR and recognize the secondary phase coupling feature of the signal. The proposed method has a good real-time performance and provides an effective method to determine the early crack fault of gear root.
\end{abstract}

\section{Introduction}

A common method for extracting the mechanical fault is assuming that vibration signals are stable with Gaussian distribution [1-3]. But the real measured signals often are nonstationary and non-Gaussian distribution. In particular, in the event of failure, signal is easy to be affected by the Gaussian noise. The gear fault signal is a typical nonlinear and nonstationary signal [3]. The machinery system usually produces sum and difference frequency components when the failure occurs. This is a nonlinear coupling phenomenon. In power spectrum, it is presented as the sum and the difference of frequency side bands $[4,5]$. For this nonlinear phenomenon, it is difficult to solve the problem fundamentally by the traditional power spectrum analysis and processing method [5]. In theory, the Gauss noise and the non-Gaussian colored noise can be completely inhibited and the phase information of the nonlinear system can be maintained by High-order cumulants (HOC) [6, 7]. Nonlinear coupling feature can be effectively detected by HOC, which provides a reliable and effective tool for the extraction of fault features.
But the HOC have high computational complexity and cost a great deal of time. As a special case of HOC, the 1.5 dimension spectrum provides an alternative because it not only retains the advantages of HOC but also has low computational complexity $[8,9]$. Empirical mode decomposition (EMD) is a new adaptive decomposition method, and the decomposed components can reconstruct the original signal, which laid a foundation for further signal processing [10]. Based on the combination of EMD and 1.5 dimension spectrum, a fault feature extraction method is proposed in this paper. And it is applied in the gearbox fault diagnosis to explore its ability of fault feature detection.

\section{Empirical Mode Decomposition Method}

Empirical mode decomposition (EMD) is also called Huang transform, which can effectively separate various frequency components of the signal from the time curve in the form of intrinsic mode function (IMF) [10]. And the original signal can be reconstructed by decomposed component. This 
method, which has been researched widely, is very suitable for nonlinear and nonstationary signal processing. IMF satisfies two conditions $[10,11]$. (1) In the entire sequence, the number of extrema (maxima plus minima) and the number of zerocrossing points is equal or differs by one. (2) At any time, the mean value of the envelope, as defined by the local maxima and the local minima, is zero. EMD is a process of cyclic decomposition; the specific steps can be described as follows: For a given signal $x(t)$, locate all the local extrema. Then fit all the extrema on the curve to construct the two envelopes $u_{0}(t)$ and $v_{0}(t)$. The mean of the upper and lower envelopes $m_{0}(t)$ is calculated as

$$
m_{0}(t)=\frac{1}{2}\left(u_{0}(t)+v_{0}(t)\right) .
$$
as

After $x(t)$ subtracts $m_{0}(t)$, the remaining part is obtained

$$
h_{1}(t)=x(t)-m_{0}(t) .
$$

Ideally, $h_{1}(t)$ should be an IMF. If $h_{1}(t)$ cannot meet the condition of LMF, taking $h_{1}(t)$ as the original signal, the corresponding upper envelope $u_{1}(t)$ and lower envelope $v_{1}(t)$ of $h_{1}(t)$ are calculated and the same interpolation scheme is reiterated to the remainder. Consider

$$
\begin{gathered}
m_{1}(t)=\frac{1}{2}\left(u_{1}(t)+v_{1}(t)\right), \\
h_{2}(t)=h_{1}(t)-m_{1}(t), \\
\vdots \\
m_{k-1}(t)=\frac{1}{2}\left(u_{k-1}(t)+v_{k-1}(t)\right), \\
h_{k}(t)=h_{k-1}(t)-m_{k-1}(t) .
\end{gathered}
$$

The above procedure is repeated till $h_{k}(t)$ conforms to the properties of IMFs described previously. $h_{k}(t)$ is the first IMF of original signal $x(t)$, called $C_{1}(t)$. Then the first $C_{1}(t)$ is subtracted by $x(t)$ to get the residue $r_{1}(t)$. Consider

$$
\begin{aligned}
C_{1}(t) & =h_{k}(t), \\
r_{1}(t) & =x(t)-C_{1}(t) .
\end{aligned}
$$

The residue $r_{1}(t)$, which contains longer-period components, is treated as new data and subjected to the same sifting process as described above. This procedure can be repeated to obtain all the subsequent $r_{j}(t)(j=1,2, \ldots, n)$. Consider

$$
\begin{gathered}
r_{1}(t)-C_{2}(t)=r_{2}(t), \\
\vdots \\
r_{n-1}(t)-C_{n}(t)=r_{n}(t) .
\end{gathered}
$$

At the end of the decomposition, $x(t)$ is represented as the sum of $n$ IMFs and a residue $r_{n}(t)$ :

$$
x(t)=\sum_{i=1}^{n} C_{i}+r_{n}
$$

In order to verify the reconfigurable property of EMD, a simulated signal is processed. Firstly, the original signal is decomposed with EMD method, and then the obtained IMFs are summed to reconstruct signal. Figure 1 shows the error between the reconstructed signal and the original signal. From these diagrams, it can be seen that the error is small between the reconstructed signal and the original signal. The amplitude of error and that of signal are different in 15 orders of magnitude. The error is computer calculation error and can be negligible. Being visible, the original signal can be reconstructed using the decomposed component, with almost no energy loss $[10,12]$, which provides a new way for noise suppression.

\section{Higher Order Statistics and Higher Order Spectrum}

Higher order statistics is a mathematical tool which is used to describe the higher order (second-order or above) statistical feature of a random process $[6,13]$. Being similar to the power spectrum of Fourier transform of autocorrelation function, multidimension Fourier transform of higher order cumulant is defined as higher order spectrum (or multispectrum) [14, 15].

For a random variable $x(t)$, the joint cumulant for the order number $r=k_{1}+k_{2}+\cdots+k_{n}$ is defined as

$$
\begin{aligned}
c_{k_{1}, k_{2}, \ldots, k_{n}} & =\left.(-j) \frac{\partial^{\prime} \psi\left(\omega_{1}, \omega_{2}, \ldots, \omega_{n}\right)}{\partial \omega_{1}^{k_{1}}, \omega_{2}^{k_{2}}, \ldots, \omega_{n}^{k_{n}}}\right|_{\omega_{1}=\omega_{2}=\cdots=\omega_{n}=0} \\
& =\left.(-j) \frac{\partial^{\prime} \Phi\left(\omega_{1}, \omega_{2}, \ldots, \omega_{n}\right)}{\partial \omega_{1}^{k_{1}}, \omega_{2}^{k_{2}}, \ldots, \omega_{n}^{k_{n}}}\right|_{\omega_{1}=\omega_{2}=\cdots=\omega_{n}=0} .
\end{aligned}
$$

Here, $\Phi\left(\omega_{1}, \omega_{2}, \ldots, \omega_{n}\right)=E\left\{\exp \left[j\left(\omega_{1} x_{1}+\omega_{2} x_{2}+\cdots+\omega_{n} x_{n}\right]\right\}\right.$.

If $x(t)$ is $k$ order stationary process of zero mean, $k$ order accumulation of this process is defined as

$$
\begin{aligned}
c_{k x} & \left(\tau_{1}, \tau_{2}, \ldots, \tau_{n}\right) \\
& =\operatorname{cum}\left(x(t), x\left(t+\tau_{1}\right), \ldots, x\left(t+\tau_{k-1}\right)\right) .
\end{aligned}
$$

Here, $\operatorname{cum}(g(x))$ is the united accumulation.

Assuming that the higher order accumulation $c_{k x}\left(\tau_{1}, \ldots, \tau_{k-1}\right)$ is absolutely summable,

$$
\sum_{\tau_{1}=-\infty}^{+\infty} \ldots \sum_{\tau_{2}=-\infty}^{+\infty}\left|c_{k x}\left(\tau_{1}, \ldots, \tau_{k-1}\right)\right|<\infty
$$

then $K$ order cumulative spectrum is defined as $k-1$ dimension Fourier transform of $k$ order cumulant:

$$
\begin{aligned}
& s_{k x}\left(\omega_{1}, \ldots, \omega_{k-1}\right) \\
& \quad=\sum_{\tau_{1}=-\infty}^{+\infty} \ldots \sum_{\tau_{2}=-\infty}^{+\infty} c_{k x}\left(\tau_{1}, \ldots, \tau_{k-1}\right) \exp \left\{-j \sum_{i=1}^{k-1} \omega_{i} \tau_{i}\right\} .
\end{aligned}
$$

Traditionally, higher order cumulant spectrum is called higher order spectrum or multispectrum; the most common 


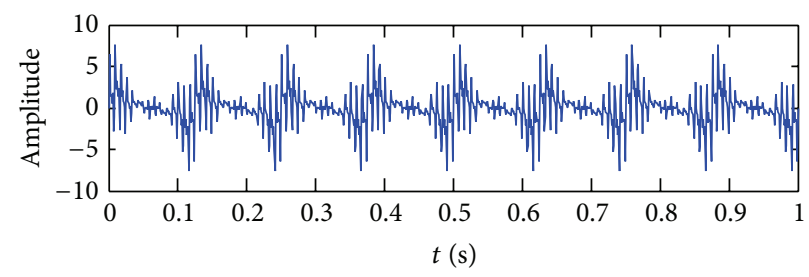

(a)
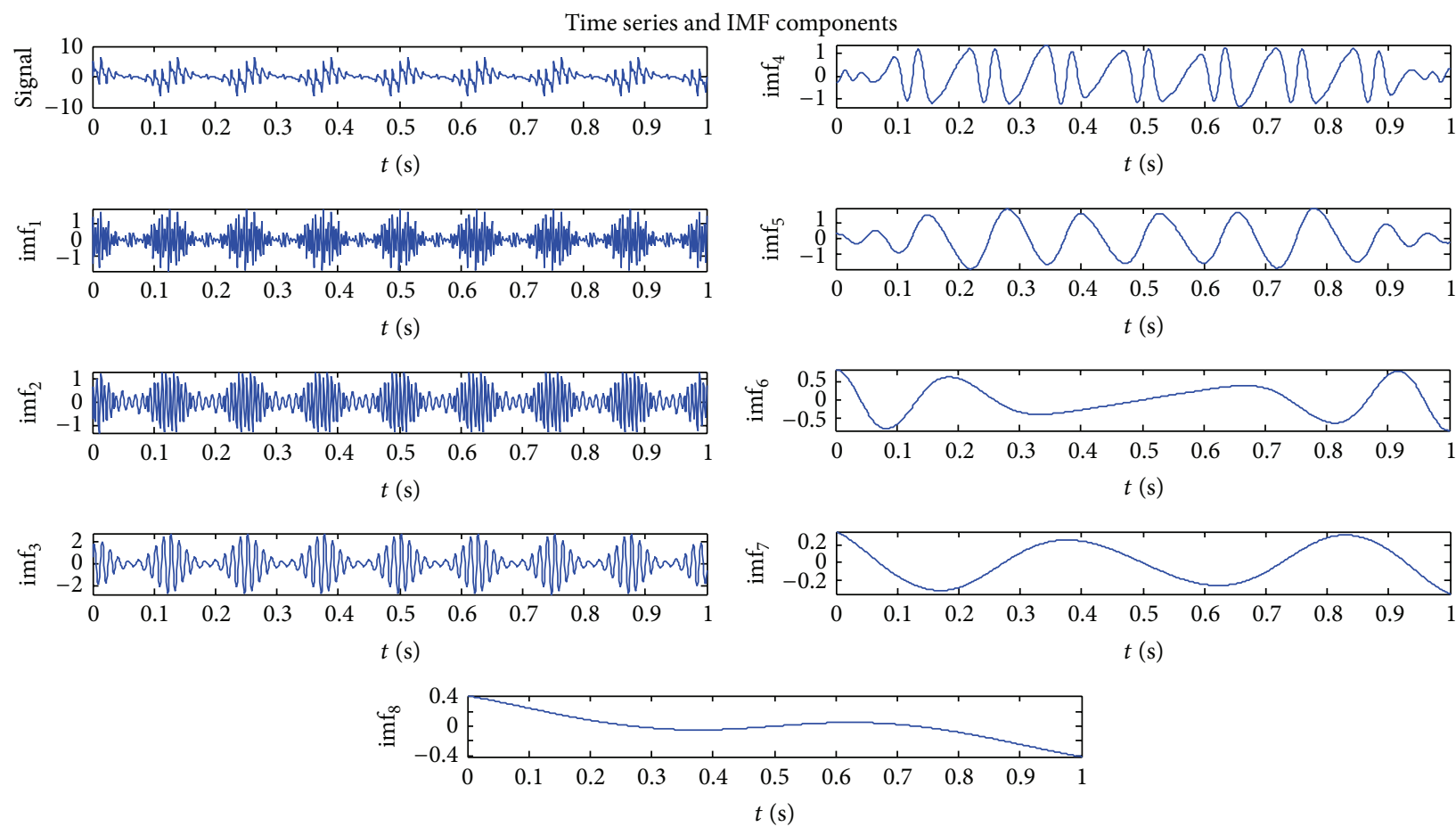

(b)

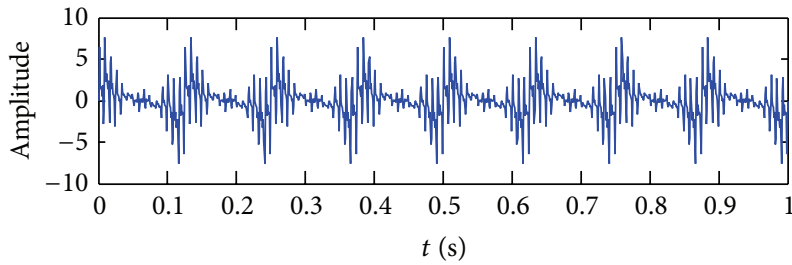

(c)

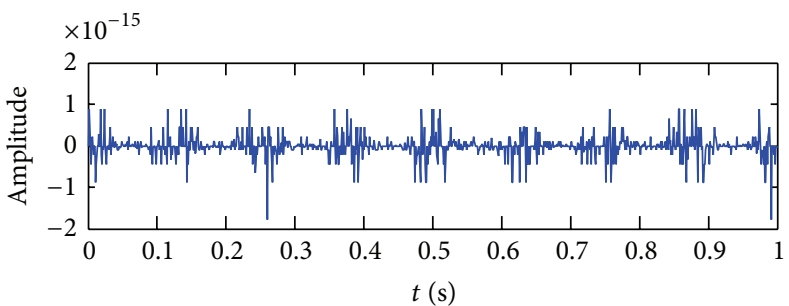

(d)

FIGURE 1: Error between the reconstructed signal and original signal. (a) Original signal. (b) EMD decomposition of original signal. (c) Reconstructed signal. (d) Error.

high order spectrum is three-order spectrum (known as double spectrum). Consider

$$
B_{x}\left(\omega_{1}, \omega_{2}\right)=\sum_{\tau_{1}=-\infty}^{+\infty} \cdots \sum_{\tau_{2}=-\infty}^{+\infty} c_{3 x}\left(\tau_{1}, \tau_{2}\right) e^{-j\left(\omega_{1} \tau_{1}+\omega_{2} \tau_{2}\right)}
$$

\section{1.5 Dimension Spectrum}

For a random variable $x(t)$, the diagonal slice of its threeorder cumulant $c_{3 x}\left(\tau_{1}, \tau_{2}\right)\left(\tau_{1}, \tau_{2}\right.$ is time delay) is denoted as $c_{3 x}(\tau, \tau)$. Then the Fourier transform of this diagonal slice is defined as 1.5 dimension power spectrum of $x(t)[8,16]$ :

$$
\begin{aligned}
C_{3 x}(\omega) & =\sum_{\tau=-\infty}^{+\infty} c_{3 x}(\tau, \tau) e^{-j \omega \tau}, \\
C_{3 x}(\omega) & =\int_{-\infty}^{+\infty} c_{3 x}(\tau, \tau) e^{-j \omega t} d \tau \\
& =\int_{-\infty}^{+\infty}\left[\int_{-\infty}^{+\infty} x(t) x^{2}(t+\tau) d t\right] e^{-j \omega t} d t .
\end{aligned}
$$


1.5 dimension spectrum holds several excellent characteristics and provides a convenient way for the signal processing [9].

(a) Supposing $x(t)$ is $n$-order real harmonic signal which has zero mean and its fundamental frequency is $\omega_{0}$, when $\left|\omega_{m}\right|<\left|\omega_{l}\right|$, the following expression was established:

$$
\begin{aligned}
C\left(\omega_{m}\right) & >C\left(\omega_{l}\right), \\
\omega_{m} & =m \omega_{0}, \quad m= \pm 1, \pm 2, \ldots, \pm n, \\
\omega_{l} & =l \omega_{0}, \quad l= \pm 1, \pm 2, \ldots, \pm n .
\end{aligned}
$$

The properties show that the fundamental frequency component of signal is strengthened when the 1.5 dimension spectrum is used to analyze harmonic signal. It provides a good method to extract the fundamental frequency component of the signal.

(b) In case that $n(t)$ is Gauss white noise with zero mean, its 1.5 dimension spectrum can be established as $C(\omega)=0$. The property indicates that the 1.5 dimension spectrum can effectively restrain the Gauss white noise.

(c) Assuming that $x(t)$ is harmonic signal, $\omega_{1}, \omega_{2}$, and $\omega_{3}$ are the three harmonic components, and $\omega_{1}>\omega_{2}>\omega_{3}$. If $\omega_{1} \neq \omega_{2}+\omega_{3}$, which means that frequency does not meet the coupling relationship, the following expression can be obtained:

$$
\begin{aligned}
& C\left(\omega_{1}\right)=0, \\
& C\left(\omega_{2}\right)=0, \\
& C\left(\omega_{3}\right)=0 .
\end{aligned}
$$

If $\omega_{1}=\omega_{2}+\omega_{3}$, which means that frequency meets the coupling relationship, the following must be determined:

$$
\begin{aligned}
& C\left(\omega_{1}\right) \neq 0, \\
& C\left(\omega_{2}\right) \neq 0, \\
& C\left(\omega_{3}\right) \neq 0 .
\end{aligned}
$$

The property suggests that three coupling harmonic terms can be extracted by 1.5 dimension spectrum and the harmonic term, which does not meet coupling relationship, can be eliminated.

1.5 dimension spectrum is a special case of the higher order spectrum. It has the ability to suppress Gauss noise and symmetrical distributed noise and has the minimum amount of computation which is similar to the amount of Fourier power spectrum in the high order spectrum [9]. The fundamental frequency component of harmonic signal can be strengthened and the nonquadratic phase coupling harmonic components can be eliminated by the 1.5 dimension spectrum. Quadratic phase coupling is a kind of nonlinear phase coupling way. For three components $c_{1}, c_{2}$, and $c_{3}$ (frequency of each component is $f_{1}, f_{2}$, and $f_{3}$, resp.; phase of each component is $\varphi_{1}, \varphi_{2}$, and $\varphi_{3}$ correspondingly), if $f_{3}=f_{1}+f_{2}$ and $\varphi_{3}=\varphi_{1}+\varphi_{2}$, then component $c_{3}$ is caused by the quadratic phase coupling of $c_{1}$ and $c_{2}$. When mechanical system failure occurs, the strong nonlinear feature is shown and quadratic phase coupling is generated by system. Traditional power spectrum inhibits all phase information. So it cannot be used to identify the quadratic phase coupling characteristic of mechanical fault signal. Bispectrum, which can be used to check the characteristics of quadratic phase coupling, retains the phase information of non-Gauss process. But the amount of computation of bispectrum is large and its estimation precision is low. In contrast, the analysis performance of bispectrum for quadratic phase coupling can be retained and the Gauss noise in signal can be inhibited completely by the 1.5 dimension spectrum [16].

\section{Methods and Steps}

Figure 2 illustrates the algorithm flow chart of fault diagnosis based on EMD-1.5 dimension spectrum. According to the above principle, the fault diagnosis method can be described as follows:

(1) In order to eliminate the interference of cross-term which is introduced by different frequency components, the fault signal $x(t)$ is decomposed by EMD to obtain a series of IMF components as $c_{1}, c_{2}, \ldots, c_{n}$.

(2) The correlation analysis is applied between the obtained IMF and the original signal, and then the pseudocomponent with small correlation coefficient is eliminated.

(3) The 1.5 dimension spectrum is calculated from the selected IMF components.

(4) The 1.5 dimension spectrum of each order of IMF is added up to get the 1.5 dimension spectrum of original signal.

(5) Finally the diagnostic results are obtained from the reconstructed 1.5 dimension spectrum.

\section{Simulated Signal Analysis}

The effectiveness of the fault diagnosis method based on EMD decomposition and 1.5 dimension spectrum will be verified by analyzing the simulated signal. Simulated signal is described as follows:

$$
\begin{gathered}
x(t)=\left[\cos \left(35 * 2 * \pi * t+\frac{\pi}{3}\right)\right. \\
\left.+\cos \left(75 * 2 * \pi * t+\frac{\pi}{6}\right)\right]^{2} \\
+\cos \left(160 * 2 * \pi * t+\frac{\pi}{4}\right) .
\end{gathered}
$$

The sampling frequency is $1 \mathrm{kHz}$; the length of data is 1000 points. The simulated signal time-domain waveforms are shown in Figure 3. According to Euler formula, the above simulated signal can be launched into the sum of some cosine signals as $70 \mathrm{~Hz}, 110 \mathrm{~Hz}, 40 \mathrm{~Hz}, 150 \mathrm{~Hz}$, and $160 \mathrm{~Hz}$. Figure 4 shows the spectrum of simulated signal from Fourier transform.

Figure 5 shows the results of the EMD decomposition of the simulated signal and the corresponding power spectrums 


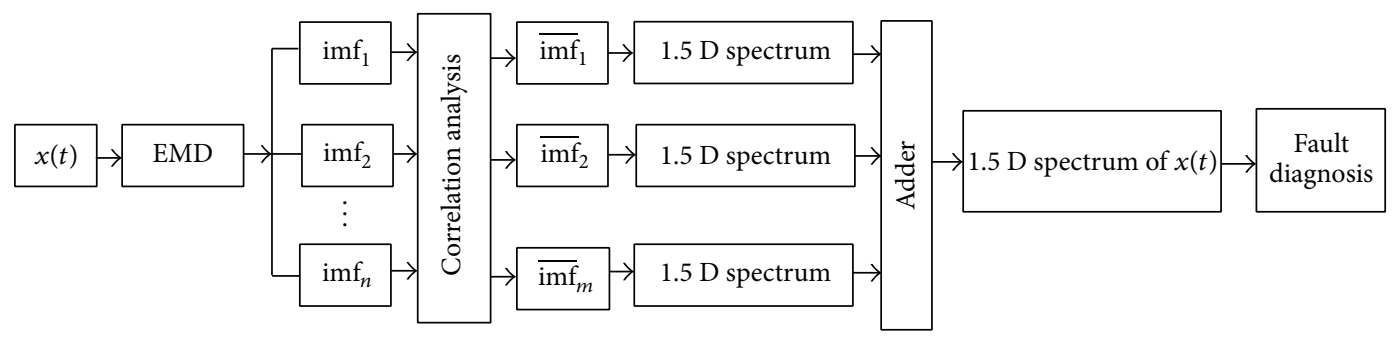

FIGURE 2: Algorithm flow chart of fault diagnosis based on EMD-1.5 dimension spectrum.

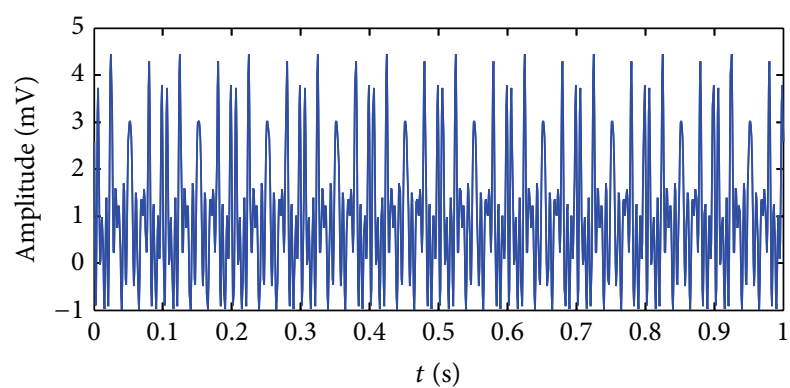

FIGURE 3: Time series of simulated signal.

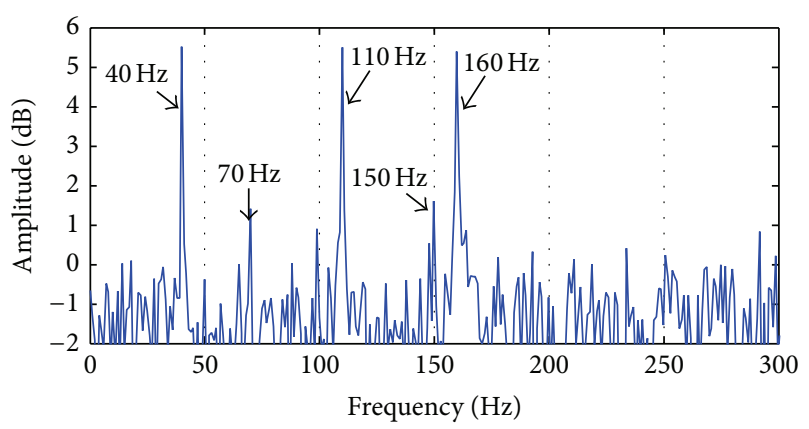

FIGURE 4: Fourier spectrum of simulated signal.

of the obtained IMFs. From the power spectrum of each IMF component, it can be seen that the decomposition process is carried out from high frequency to low frequency, which illustrates that EMD has the characteristics of multiresolution analysis. The components "imf." " $\mathrm{imf}_{5}$ " are the dominant components, and "Residual" is the remaining amount or a weak item because its amplitude is 1/200 of original signal. Thus, the EMD is an adaptive process of decomposition from high frequency to low frequency.

Calculating the correlation coefficients between each IMF and the original signal, respectively (in other words, the correlation coefficient between "imf. "and the original signal is calculated, and then the correlation coefficient between "imf " and the original signal is calculated, and so on), they are $0.761,0.753,0.624,0.617,0.178$, and 0.162 . It can be seen that "imf." " imf 4 " have much larger correlation coefficient than the following two components. Their value is not in the same order of magnitude. Thus, the components after "imf 4 can be deduced as the false component. In the analysis of original signal, the 1.5 dimension spectrum of "imf " " $\mathrm{imf}_{4}$ " is calculated. And 1.5 dimension spectrum of original signal can be obtained after superposition. Figure 6 shows the 1.5 dimension spectrum of simulated signal.

Figure 4 is compared with Figure 6. In the Fourier spectrum shown in Figure 4, the frequency components of the simulated signal, respectively, $40 \mathrm{~Hz}, 70 \mathrm{~Hz}, 110 \mathrm{~Hz}$, $150 \mathrm{~Hz}$, and $160 \mathrm{~Hz}$, can be detected. Gauss white noise exists throughout the frequency band, and its amplitude is large. In the 1.5 dimension spectrum shown in Figure 6, the frequency components which meet nonlinear coupling relationship are $40 \mathrm{~Hz}, 70 \mathrm{~Hz}, 110 \mathrm{~Hz}$, and $150 \mathrm{~Hz}$. The frequency component $160 \mathrm{~Hz}$, which is not in the coupling, is eliminated. This also verifies the property of the 1.5 dimension spectrum discussed above.

In order to further verify the ability of suppressing of Gauss white noise, Figure 7 shows the 1.5 dimension spectrum and Fourier spectrum of noised signals which are from different SNR: $10 \mathrm{~dB}, 0 \mathrm{~dB}$, and $-10 \mathrm{~dB}$. In the three situations of spectrum, even though in the case of $\mathrm{SNR}=0 \mathrm{~dB}$, the 1.5 dimension spectrum can still accurately extract the peak value of 4 components. The spectrum line is relatively smooth, the frequency components are pure, and the peak feature is clear after noise reduction. After adding the noise with $\mathrm{SNR}=-10 \mathrm{~dB}$, which is a strong interference, the peak values of $40 \mathrm{~Hz}$ and $110 \mathrm{~Hz}$ frequency components are still clearly presented. Comparing the Fourier spectrum with the 1.5 dimension spectrum of simulated signal, it can be drawn that the signal extraction ability of Fourier spectrum is weaker under the condition of above three kinds of noise. In particular, when $\mathrm{SNR}=-10 \mathrm{~dB}$, the peak of Fourier spectrum of useful signal is almost submerged by that of nose. Thus it can be seen that 1.5 dimension spectrum can effectively remove the Gauss noise and reduce effect of noise and has a better effect than Fourier spectrum.

Table 1 compares the running time of Fourier spectrum, 1.5 dimension spectrum, and bispectrum under different SNR circumstances. Spectrum estimation processions of three kinds of method are similar. In Table 1, the speed of spectrum estimation based on Fourier transform is the fastest in the case of same data length and SNR. Although the speed of the 1.5 dimension spectrum is slower than that of the Fourier method, the computation time is significantly shorter than that of the bispectrum. The efficiency has been improved by almost 3 times of magnitude. The running time of the bispectrum is increased from $1.86 \mathrm{~s}$ to $3.87 \mathrm{~s}$ when the SNR 

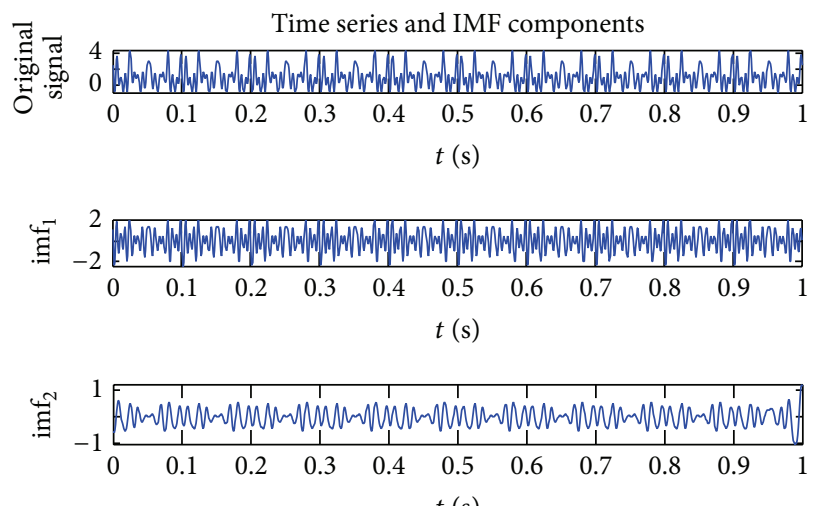

$t(\mathrm{~s})$
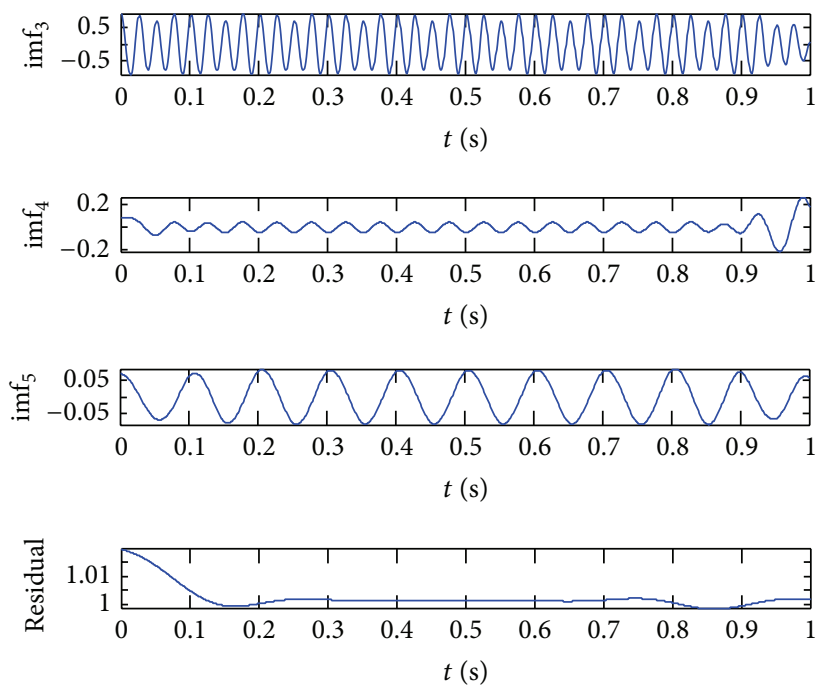
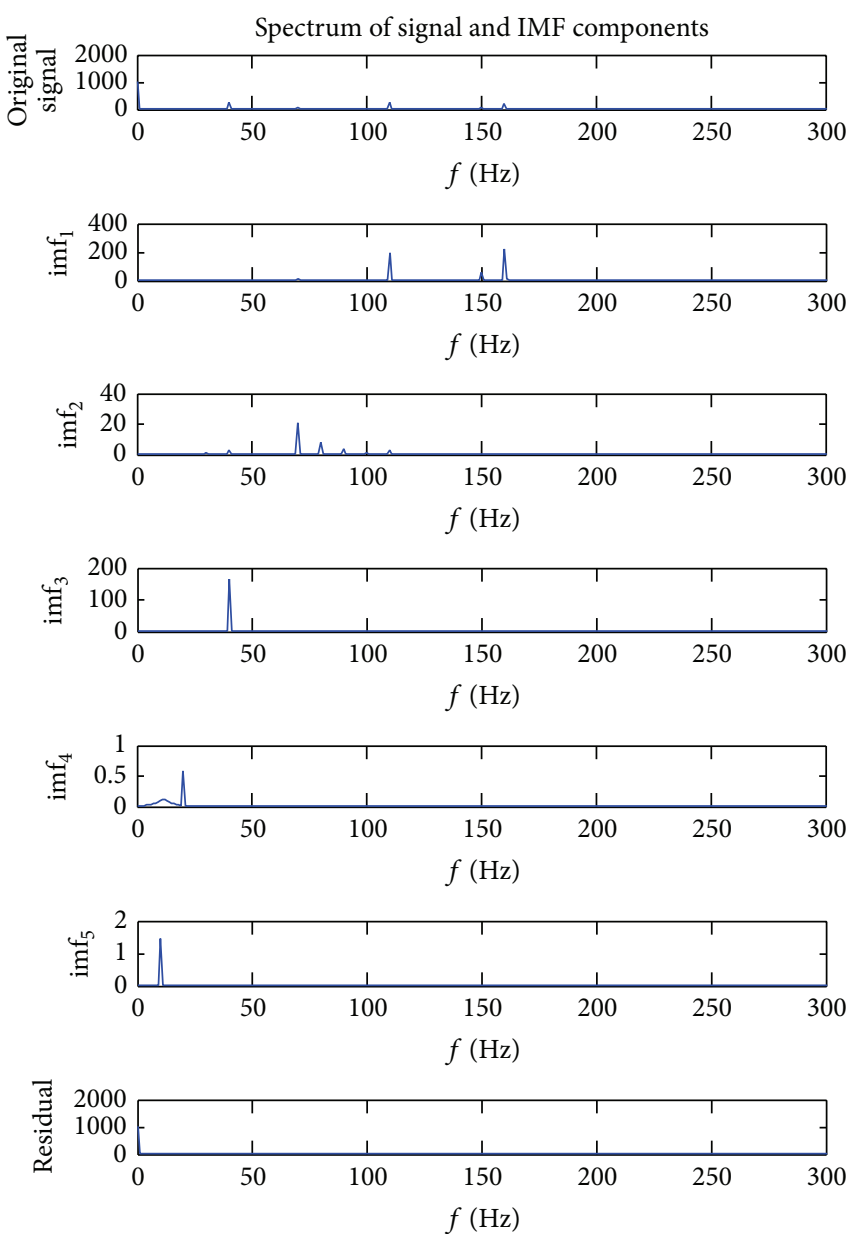

FIGURE 5: EMD decomposition and spectrum of IMFs of simulated signal.

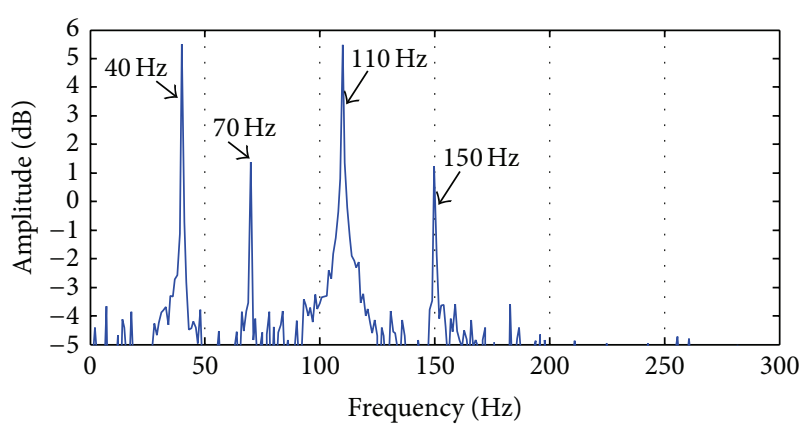

FIGURE 6: EMD-1.5 dimension spectrum of simulated signal.

is decreased from $20 \mathrm{~dB}$ to $-20 \mathrm{~dB}$. Meanwhile the running time of the 1.5 dimension spectrum is only increased from $0.542 \mathrm{~s}$ to $0.968 \mathrm{~s}$, and the increase is small.

From the above simulation experiments, we come to a conclusion that the 1.5 dimension spectrum can effectively extract the nonlinear coupling features of the signal while restraining the Gauss white noise and it is faster than other higher order spectrum. 1.5 dimension spectrum is suitable for the real-time feature extraction of fault signal under mass data and low SNR condition.
TABLE 1: Run-time comparison of three methods under different SNR conditions.

\begin{tabular}{lcccc}
\hline $\begin{array}{l}\text { Sampling } \\
\text { points }\end{array}$ & SNR/dB & $\begin{array}{c}\text { Fourier } \\
\text { spectrum }\end{array}$ & $\begin{array}{c}\text { Running time/s } \\
\text { 1.5 dimension } \\
\text { spectrum }\end{array}$ & Bispectrum \\
\hline \multirow{4}{*}{1024} & -20 & 0.819 & 0.968 & 3.87 \\
& -10 & 0.736 & 0.975 & 2.93 \\
& 0 & 0.489 & 0.761 & 2.07 \\
& 10 & 0.314 & 0.653 & 1.97 \\
& 20 & 0.205 & 0.542 & 1.86 \\
\hline
\end{tabular}

\section{Measured Signal}

In order to further verify the effectiveness of the proposed method in the fault feature extraction of gears, the gear with gear tooth crack fault is tested and analyzed. A comprehensive fault simulation test bench is used to do gear fault diagnosis test. The test bench is composed of motor, a mechanical transmission device, sensor, the hardware circuit, computer, and related software. Figure 8 shows the test bench. The number of teeth of driving wheel is $Z_{1}=10$ and motor speed is $f_{r 1}=720 \mathrm{rpm}$. The number of teeth of driven wheel 

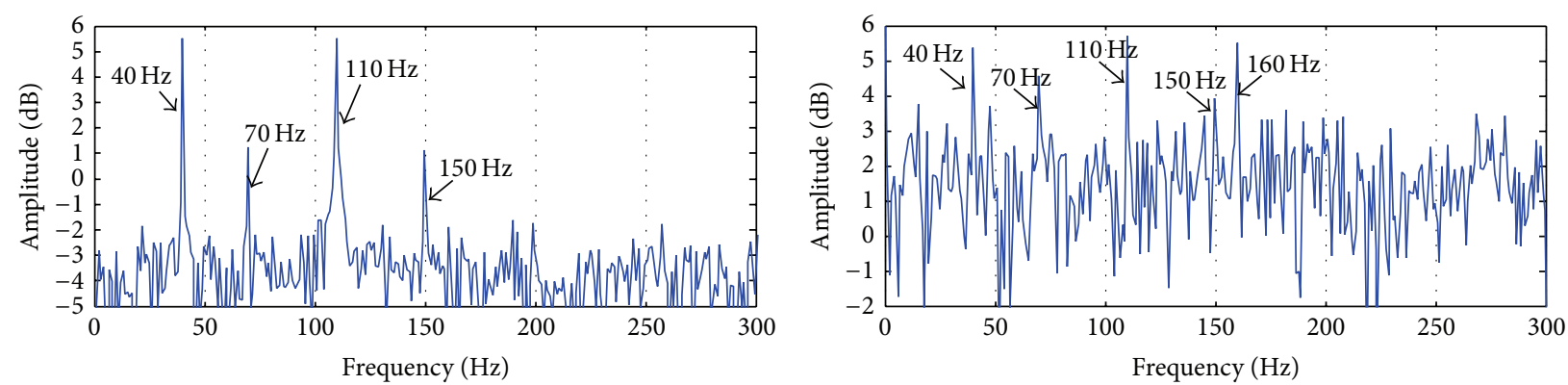

(a)
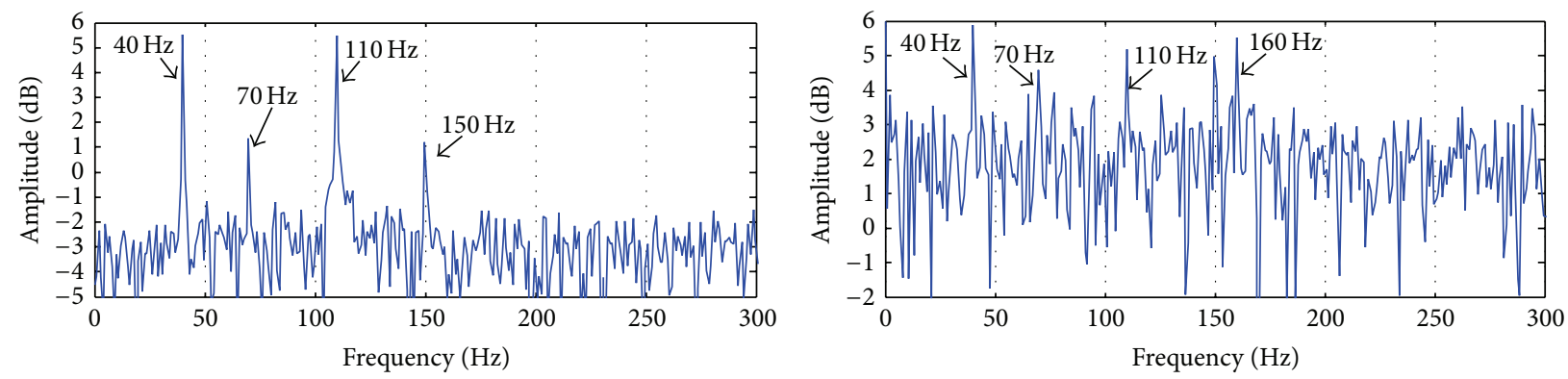

(b)
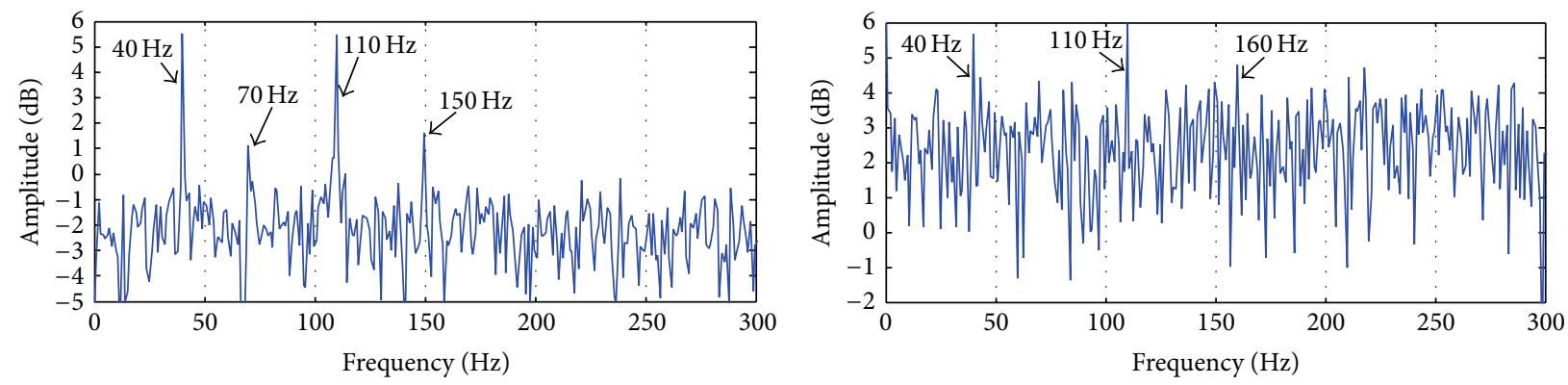

(c)

FIGURE 7: EMD-1.5 dimension spectrum (left) and Fourier spectrum (right) of simulated signal with different SNR. (a) SNR = $10 \mathrm{~dB}$. (b) SNR $=0 \mathrm{~dB} .(\mathrm{c}) \mathrm{SNR}=-10 \mathrm{~dB}$.

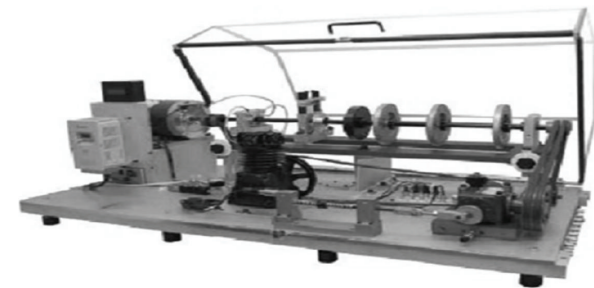

FIGURE 8: Comprehensive fault simulation test bench.

gear is $Z_{2}=15$, module is $m=2 \mathrm{~mm}$, rotating frequency of gear axis is $f_{r 2}=480 \mathrm{rpm}$, meshing frequency is $f_{z}=$ $120 \mathrm{~Hz}$, and sampling frequency is $1 \mathrm{kHz}$. A gear with defects has been considered and a small crack to one gear has been added. In the gear, a small groove, with $0.1 \mathrm{~mm}$ width and $3 \mathrm{~mm}$ depth, is processed to simulate tooth crack. Figure 9 shows the time-domain waveform of the gearbox vibration signal. It can be seen that the periodic impulse signals are almost drowned in the strong background noise, and the

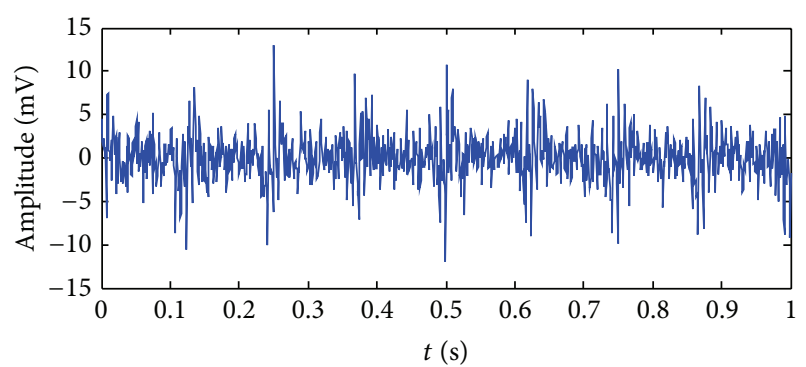

FIgURE 9: Time series of measured signal.

time-domain waveform is very complex. Figure 10 illustrates the corresponding Fourier spectrum of the signal. In the spectrum, the specific features of the signal are difficult to be distinguished, so it is difficult to extract the fault signal.

The fault signal is decomposed by EMD method. The IMF components and their corresponding power spectrum are shown in Figure 11. In the figure, it can be seen that the 


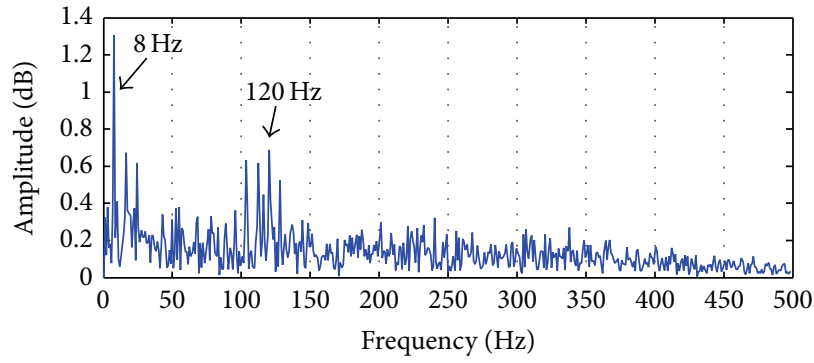

FIGURE 10: Fourier spectrum of measured signal.
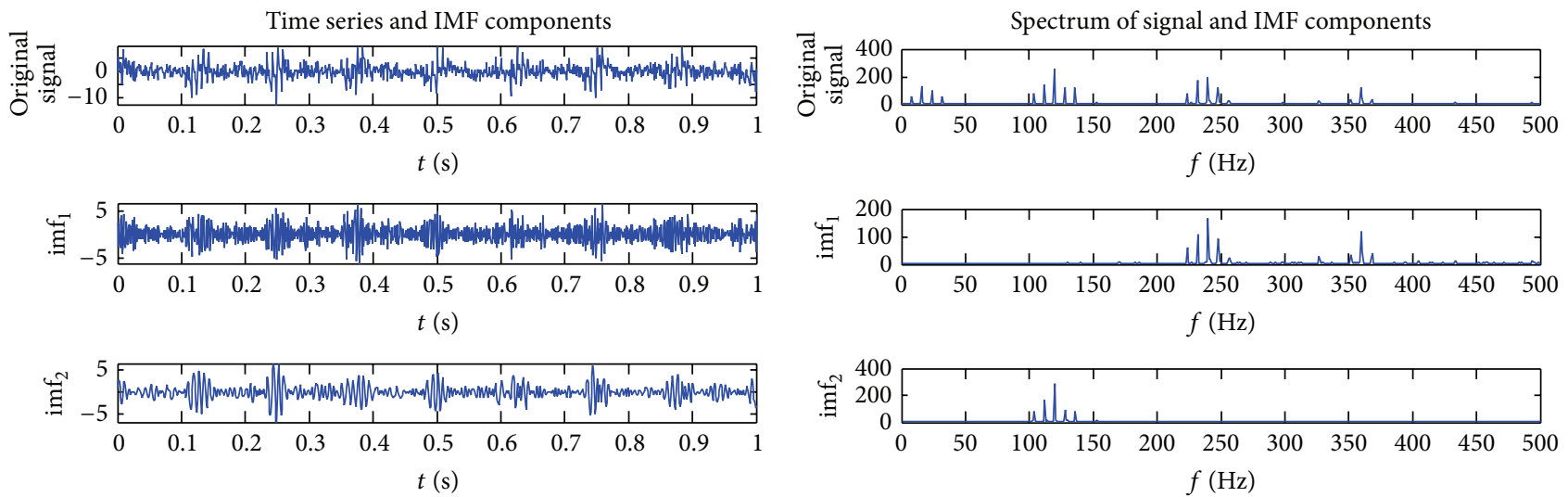

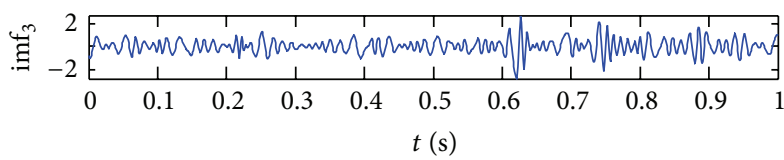
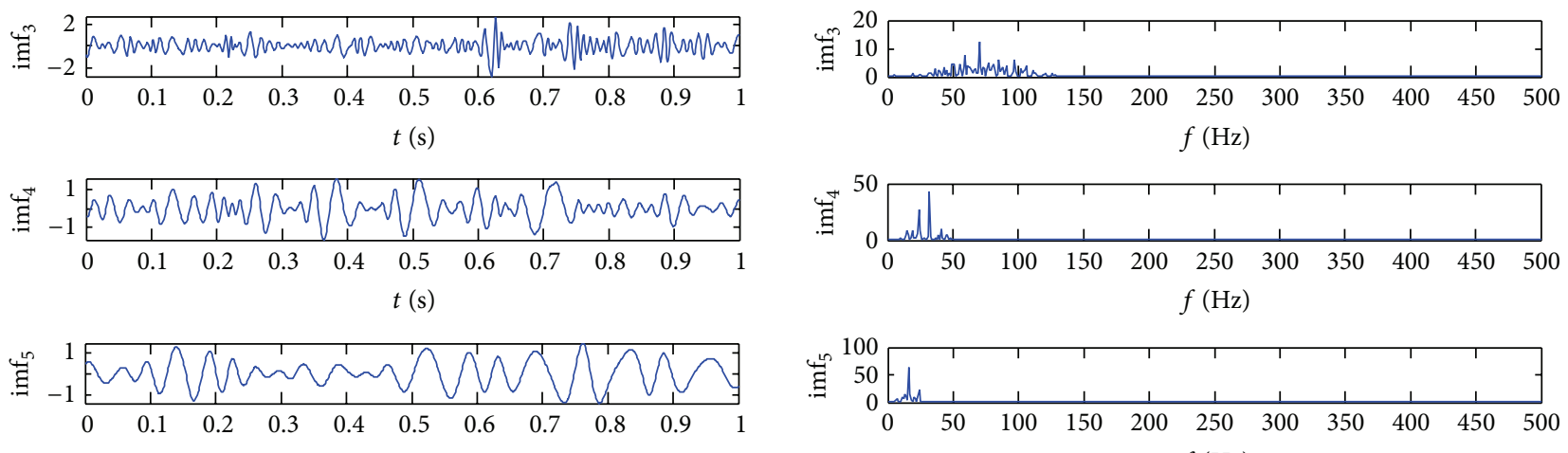
$t(\mathrm{~s})$
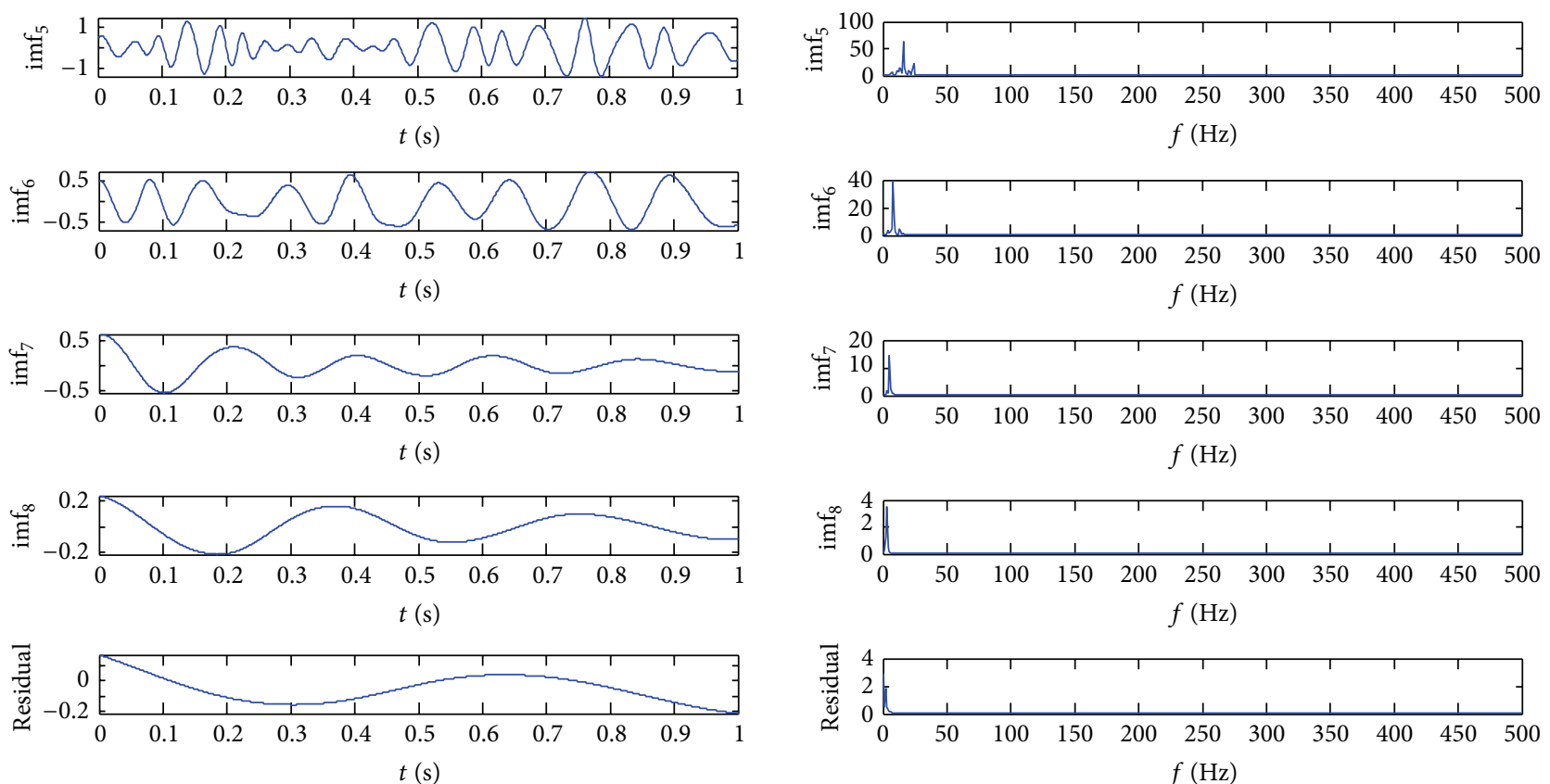

FIGURE 11: EMD decomposition and spectrum of IMFs of measured signal. 


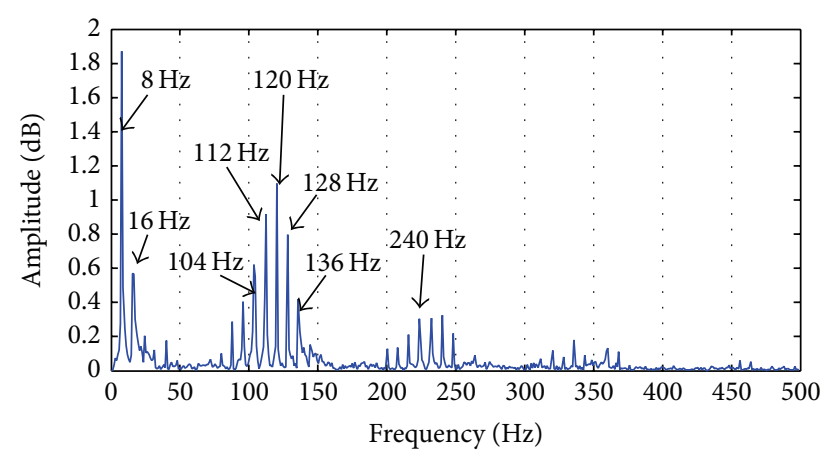

FIGURE 12: EMD-1.5 dimension spectrum of measured signal.

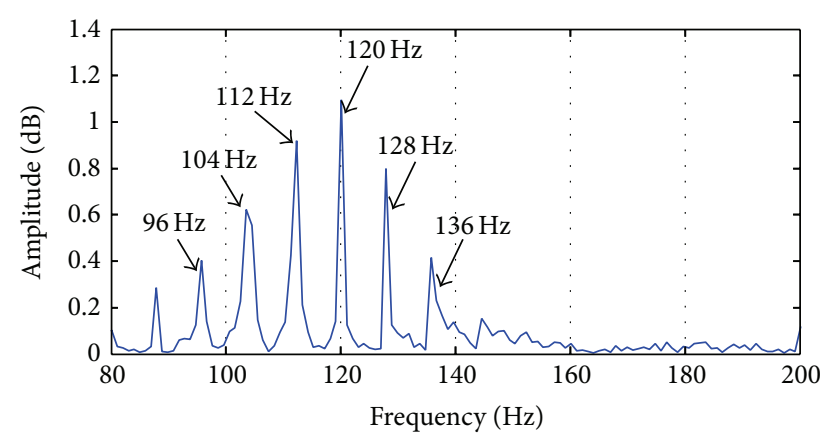

FIGURE 13: Partial enlarged drawing of EMD-1.5 dimension spectrum of measured signal $(80 \mathrm{~Hz}-200 \mathrm{~Hz})$.

signal is decomposed into 9 layers from high frequency to low frequency. The correlation coefficients between each component and the signal are calculated: they are $0.711,0.704,0.484$, $0.701,0.714,0.632,0.556$, and 0.521 . The IMF component, whose correlation coefficient is more than 0.5 , is chosen to calculate 1.5 dimension spectrum. After superposition, the spectrums of fault signal are shown in Figure 12. In order to observe the meshing frequency and its edge frequency band, Figure 12 is amplified locally. Figures 14 and 13 show the spectrum line of the part frequency range.

In Figure 12, it can be clearly seen that there are obvious spectrum lines in $8 \mathrm{~Hz}, 16 \mathrm{~Hz}, 24 \mathrm{~Hz}$, and $32 \mathrm{~Hz}$, which are very close to the calculated output rotational frequency $(8 \mathrm{~Hz})$ and its harmonic frequency. In Figure 13, it is clear that there is a clear spectrum line at $120 \mathrm{~Hz}$, and the meshing frequency $(120 \mathrm{~Hz})$ is very close to the theoretical value. There are obvious spectrum lines in $104 \mathrm{~Hz}, 112 \mathrm{~Hz}, 120 \mathrm{~Hz}$, $128 \mathrm{~Hz}$, and $136 \mathrm{~Hz}$. The distance between these frequencies is very close to the output shaft rotating frequency, which illustrates that the meshing frequency is modulated by the rotating frequency. In Figure 14, it is clear that there are obvious spectrum lines in $224 \mathrm{~Hz}, 232 \mathrm{~Hz}, 240 \mathrm{~Hz}, 248 \mathrm{~Hz}$, and $256 \mathrm{~Hz}$. The distance between these frequencies is also very close to the output shaft rotating frequency in theory. $240 \mathrm{~Hz}$ is very close to the two doubling frequency $(240 \mathrm{~Hz})$ of the meshing frequency calculated in theory, which shows that the two doubling frequency of the meshing frequency is modulated by the rotating frequency. The same feature is also generated at the $360 \mathrm{~Hz}$ frequency point. From above

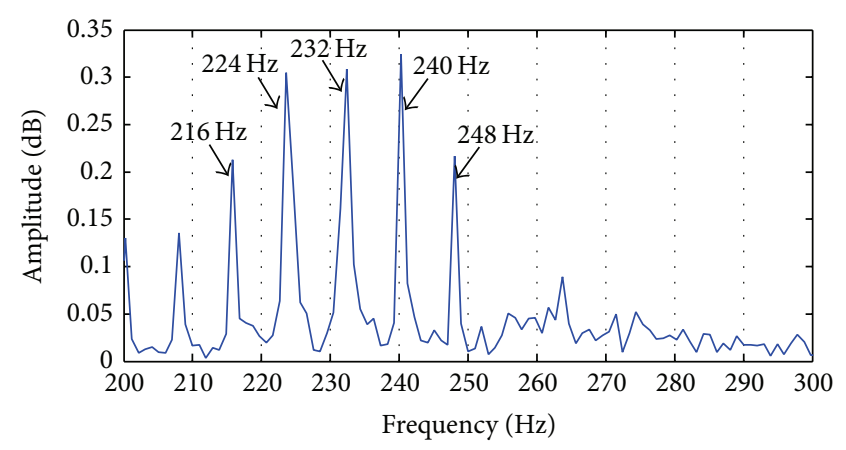

Figure 14: Partial enlarged drawing of EMD-1.5 dimension spectrum of measured signal $(200 \mathrm{~Hz}-300 \mathrm{~Hz})$.

result, we come to a conclusion that the output shaft gears have faults, which meet the actual fault conditions.

\section{Conclusion}

Combining the advantage of empirical mode decomposition and 1.5 dimension spectrum, a united mechanical fault diagnosis method is proposed. Simulated and measured signal analysis shows that EMD decomposition is a complete adaptive decomposition process for signal. And the decomposed IMF can be used to reconstruct the original signal, which lays the foundation for the selection of IMF with high correlation degree. 1.5 dimension spectrum is a special case of the high order spectrum. It has the ability to suppress Gauss noise and symmetrical distributed noise and has the minimum amount of computation which is similar to the amount of power spectrum. The analysis performance of bispectrum for quadratic phase coupling can be retained by 1.5 dimension spectrum. Based on empirical mode decomposition and 1.5 dimension spectrum, the nonlinear coupling characteristics can be extracted and the Gauss white noise can be effectively suppressed by the proposed method; EMD-1.5 dimension spectrum method provides the conditions for accurately extracting fault feature information.

\section{Conflict of Interests}

The authors declare that there is no conflict of interests regarding the publication of this paper.

\section{Acknowledgments}

The authors wish to acknowledge the assistance and support of all those who contributed to their effort to enhance and develop the described system. The authors express their appreciation for the financial support provided by the National Natural Science Foundation of China (Project no.: 41304098), Hunan Provincial Natural Science Foundation of China (Project no.: 12JJ4034), Young Scientific Research Fund of Hunan Provincial Education Department, PRC (Project no.: 13B076), Fund of the 11th Five-Year Plan for Key Construction Academic Subject (Optics) of Hunan Province, 
Optoelectronic information technology Hunan Province Talent training base between School and enterprise, and Hunan Province Key Laboratory of Photoelectric Information Integration and Optical Manufacturing Technology.

\section{References}

[1] W. A. Gardner and C. M. Spooner, "The cumulant theory of cyclostationary time-series, part I: foundation," IEEE Transactions on Signal Processing, vol. 42, no. 12, pp. 3387-3408, 1994.

[2] S. Serhat and A. Emine, "Feature extraction related to bearing damage in electric motors by wavelet analysis," Journal of the Franklin Institute, vol. 340, no. 2, pp. 125-134, 2003.

[3] H. Li, Y. Zhang, and H. Zheng, "Hilbert-Huang transform and marginal spectrum for detection and diagnosis of localized defects in roller bearings," Journal of Mechanical Science and Technology, vol. 23, no. 2, pp. 291-301, 2009.

[4] L. Shen, X. Zhou, L. Liu, and F. Yang, "Application of morphological wavelet de-noising in extracting gear fault feature," Transactions of the Chinese Society of Agricultural Machinery, vol. 41, no. 4, pp. 217-221, 2010.

[5] J. Lin and L. Qu, "Feature extraction based on Morlet wavelet and its application for mechanical fault diagnosis," Journal of Sound and Vibration, vol. 234, no. 1, pp. 135-148, 2000.

[6] W. Y. Liu, B. P. Tang, and R. X. Chen, "A fault identification method based on high-order spectral analysis of diagonal slices," Mechanical Science and Technology for Aerospace Engineering, vol. 29, no. 3, pp. 281-284, 2010.

[7] J. Cui, Y. R. Wang, and Q. Liu, “The technique of power electronic circuit fault diagnosis based on higher-order spectrum analysis and support vector machines," Proceedings of the CSEE, vol. 27, no. 10, pp. 62-66, 2007.

[8] L. Chen, Y. Y. Zi, Z. J. He, and W. Cheng, "Research and application of ensemble empirical mode decomposition principle and 1.5 dimension spectrum method," Journal of Xian Jiaotong University, vol. 43, no. 5, pp. 94-98, 2009.

[9] Y. S. Zhang, S. X. Wang, and S. B. Li, "1.5 Dimension spectrum approach to quadratic phase coupling estimstion," Acta Electronica Sinica, vol. 24, no. 4, pp. 109-112, 1996.

[10] N. E. Huang, M. C. Wu, S. R. Long et al., "A confidence limit for the empirical mode decomposition and Hilbert spectral analysis," The Royal Society of London-Series A: Proceedings, vol. 459, no. 2037, pp. 2317-2345, 2003.

[11] N. E. Huang, Z. Shen, S. R. Long et al., "The empirical mode decomposition and the Hilbert spectrum for nonlinear and non-stationary time series analysis," The Royal Society of London-Series A: Proceedings, vol. 454, no. 1971, pp. 903-995, 1998.

[12] J.-H. Cai, J.-T. Tang, X.-R. Hua, and Y.-R. Gong, "An analysis method for magnetotelluric data based on the Hilbert-Huang transform," Exploration Geophysics, vol. 40, no. 2, pp. 197-205, 2009.

[13] D. R. Veils and T. J. Ulrych, "Simulated annealing wavelet estimation via fourth-order cumulant matching," Geophysics, vol. 61, no. 6, pp. 1939-1948, 1996.

[14] C. M. Spooner and W. A. Gardner, "The cumulant theory of cyclostationary time-series. II. Development and applications," IEEE Transactions on Signal Processing, vol. 42, no. 12, pp. 34093429, 1994.
[15] J.-H. Cai, W.-W. Hu, Z.-Y. Ren, and L.-Y. Lei, "Magnetotelluric data processing and simulation based on higher-order statistics," Journal of Central South University, vol. 41, no. 4, pp. 15561560, 2010.

[16] Y. Y. Fan, B. Q. Tao, and K. Xing, "Feature extraction of shipradiated noise by 1.5 dimension spectrum," Acta Acustica, vol. 27, no. 1, pp. 71-76, 2002. 


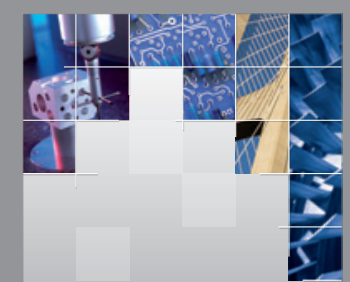

\section{Enfincering}
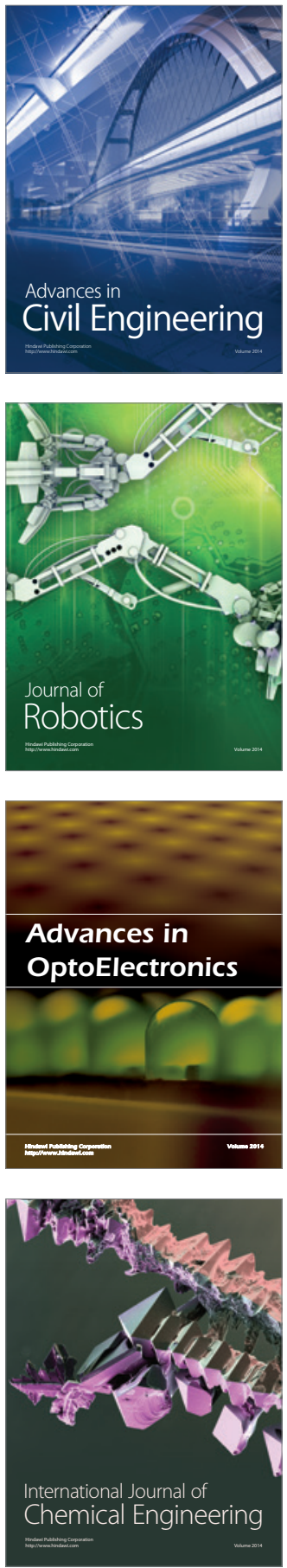

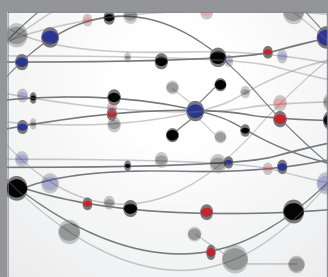

The Scientific World Journal

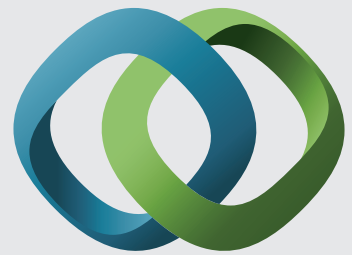

\section{Hindawi}

Submit your manuscripts at

http://www.hindawi.com
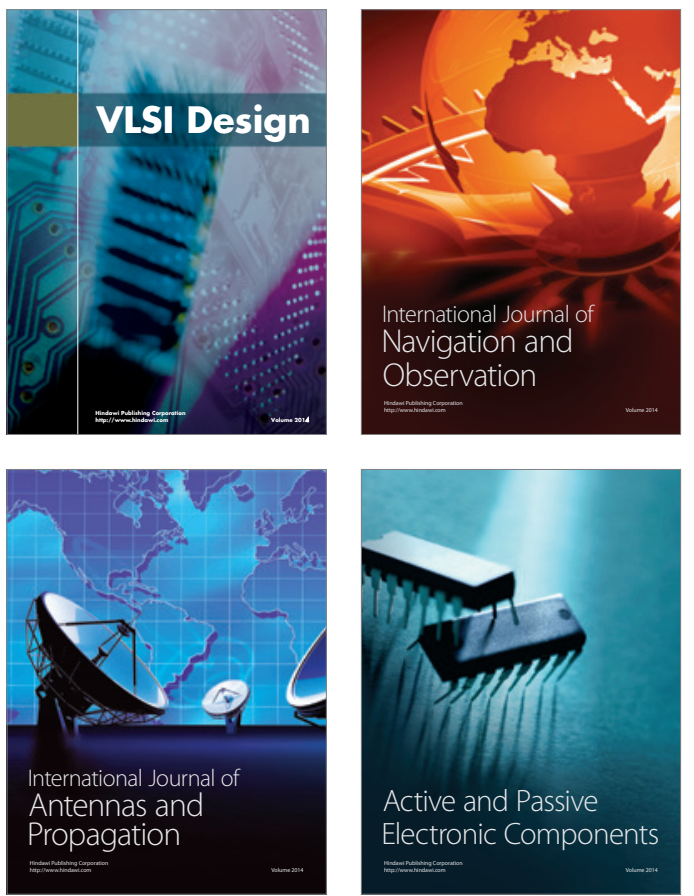
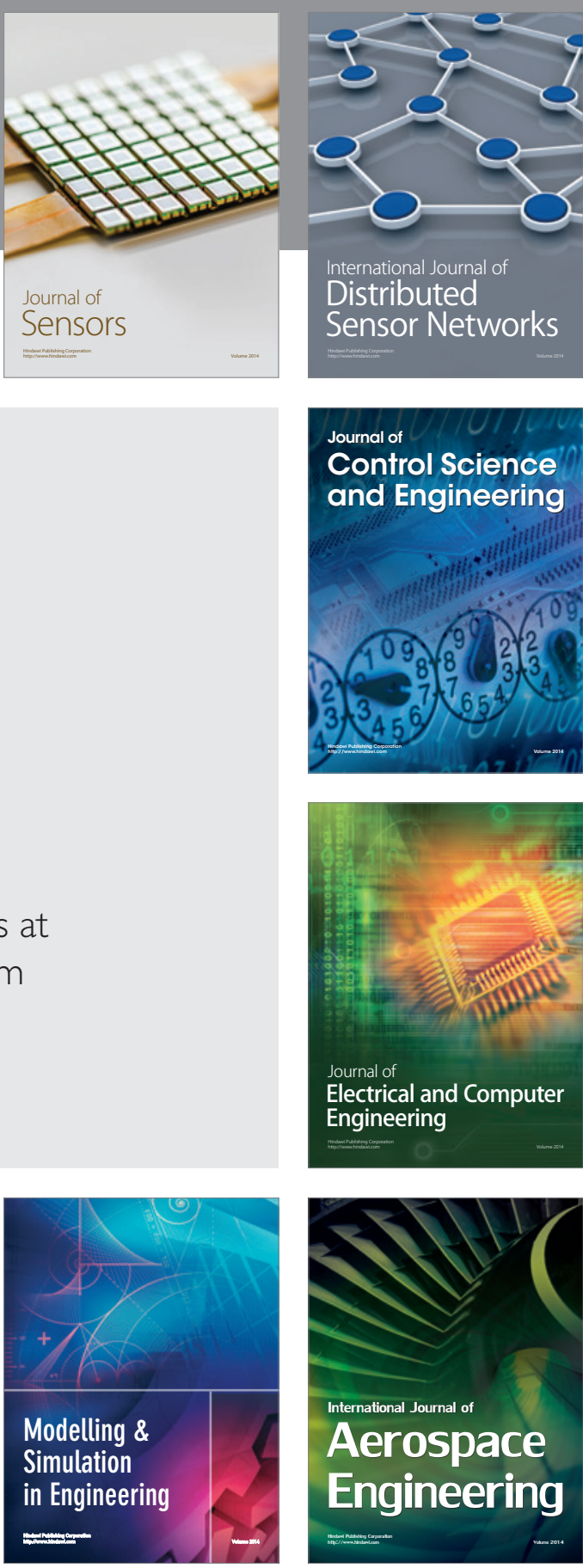

International Journal of

Distributed

Sensor Networks

Journal of

Control Science

and Engineering
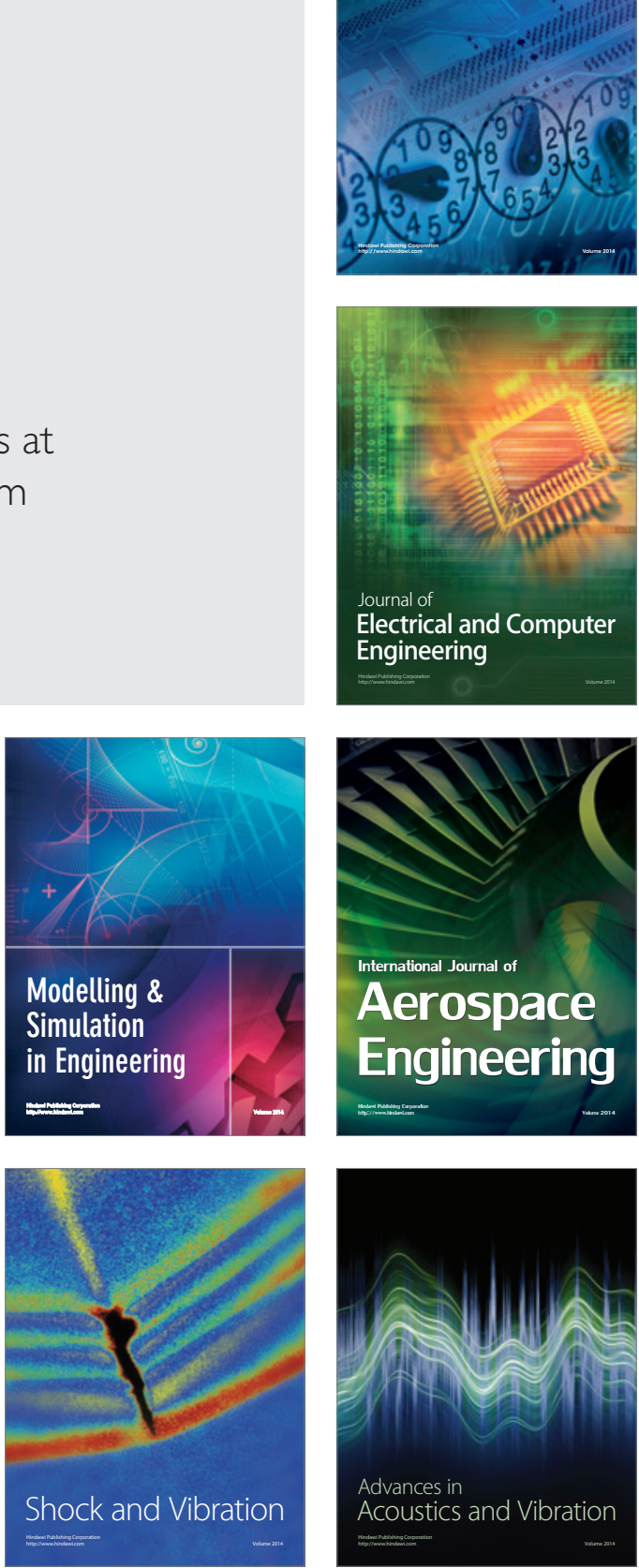